\title{
Cáncer anaplásico de tiroides de manejo quirúrgico*
}

\author{
Drs. ADRIANA LOBOS M. ${ }^{1,2}$, Ints. DIEGO VILLAGRÁN R. ${ }^{3}$, \\ CAROLINA OPAZO T. ${ }^{3}$, FELIPE CARDEMIL M. ${ }^{3}$ \\ 1 Escuela de Medicina, Universidad de Valparaíso, Valparaíso, Chile. \\ 2 Servicio de Cirugía Hospital Dr. Gustavo Fricke, Viña del Mar, Chile. \\ 3 Interno Escuela de Medicina, Universidad de Valparaíso, Chile.
}

\begin{abstract}
Anaplastic thyroid carcinoma. review of six patients

Background: Anaplastic Thyroid Carcinoma corresponds 2 to $5 \%$ of all thyroid cancers. It affects mainly women in the sixth or seventh decade of life, appearing as a hard, fast growing cervical mass that is adhered to surrounding structures. Treatment includes surgery, chemotherapy and radiotherapy. Aim: to report a series of patients with anaplastic thyroid carcinoma. Patients and Methods: Review of medical records of patients with anaplastic thyroid cancer operated between 2002 and 2008. Results: The records of six patients aged 46 to 82 years (four males), were retrieved. A bilateral total thyroidectomy was performed in four patients. Two patients required tracheostomy during the postoperative period. Mean hospital stay was six days. None received neoadjuvant or adjuvant radio-chemotherapy. Three patients died within one month of the operation. The rest died at 115, 184 and 283 days after surgery. Conclusions: All these patients were diagnosed in advanced stages of the disease, a fact that can explain the dismal evolution observed.
\end{abstract}

Key words: Anaplastic thyroid cancer, thyroidectomy, survival.

\section{Resumen}

Introducción: El Cáncer Anaplásico de Tiroides corresponde al 2 a 5\% del total de cánceres tiroideos. Afecta a mujeres en la sexta o séptima década de la vida, presentándose como masa cervical pétrea de crecimiento rápido, adherida a planos profundos. El tratamiento incluye cirugía, quimioterapia y radioterapia, siendo de elección el tratamiento multimodal. Objetivo: Conocer los resultados de una serie de casos manejados quirúrgicamente en el Hospital Dr. Gustavo Fricke de Viña del Mar. Pacientes y Método: Estudio de serie de casos de Cáncer Anaplásico de Tiroides tratados exclusivamente con cirugía entre los años 2002 y 2008. Se registraron características generales, técnica operatoria, complicaciones, uso de otras terapias y sobrevida. Resultados: Se manejaron 6 pacientes, cuatro hombres y dos mujeres. El promedio de edad fue de 59 años. La estadía hospitalaria promedio fue de 6 días. Se realizó tiroidectomía total bilateral en 4 pacientes. Dos pacientes requirieron traqueostomía durante el postoperatorio. En ninguno de ellos se realizó radioquimioterapia neoadyuvante o adyuvante. La sobrevida promedio fue de 108 días. Discusión:

*Recibido el 21 de Mayo de 2009 y aceptado para publicación el 22 de Junio de 2009.

Correspondencia: Dra. Adriana Lobos M.

Alvarez 1532, Viña del Mar, Chile.

E-mail: alobosm@gmail.com 
Destaca en esta serie el diagnóstico en etapas avanzadas de la enfermedad, lo cual determina la imposibilidad de realizar terapia multimodal y la sobrevida observada. Por lo anterior, es de importancia la sospecha y la derivación inmediata para el manejo especializado, aumentando así la posibilidad del uso de terapia multimodal con mejores resultados en términos de sobrevida. Se observó una sobrevida menor a la reportada en la literatura.

Palabras clave: Cáncer anaplásico de tiroides, tratamiento quirúrgico, sobrevida.

\section{Introducción}

El Cáncer Anaplásico de Tiroides (CAT) constituye el 2 al 5\% del total de cánceres tiroideos, siendo responsable del 40 al 50\% del total de muertes por esta patología ${ }^{1-3}$. Su frecuencia varía según el lugar geográfico; en Israel corresponde al 7,5\% mientras que en algunas regiones de Italia es un $1 \%{ }^{4}$. En Chile, este dato se desconoce. Afecta principalmente a mujeres entre la sexta y séptima décadas de la vida, correspondiendo éstas al 55 a $75 \%$ de los casos $^{3}$. Algunos autores plantean que el CAT se origina a partir de la desdiferenciación de un cáncer papilar o folicular de tiroides ${ }^{5,6}$. La incidencia del CAT ha disminuido de un $16 \%$ a un $8 \%$ durante los últimos años debido a un aumento en la detección precoz de los cánceres papilar y folicular ${ }^{2,7}$.

Molecularmente, en el CAT se genera una sobreexpresión de genes involucrados en el control de la progresión del ciclo celular y segregación cromosómica, como PLK1 (Polo-likekinase 1) y TTK ( $\mathrm{T}$ celltyrosinekinase), dos proteínas kinasas involucradas en la regulación del ciclo celular ${ }^{8}$.

La presentación clínica del CAT corresponde a una masa cervical de crecimiento rápido ${ }^{9}$, pétrea, y adherida a planos profundos. A menudo, estos pacientes se presentan con una enfermedad localmente avanzada, con síntomas de compresión e invasión local como disfagia, disnea y disfonía ${ }^{10,11}$. En el $40 \%$ de los casos existen linfadenopatías, y en el $43 \%$ al $52 \%$ de los pacientes metástasis a distancia ${ }^{11,12}$, siendo las más frecuentes pulmonares $(42 \%)$, huesos $(32 \%)$ y cerebro $(9 \%)^{9}$.

Se reportan a continuación los resultados de 6 casos de CAT manejados quirúrgicamente, describiendo características, tipo de manejo y sobrevida.

\section{Material y Método}

Se realizó un estudio de serie de casos para el que se consideraron pacientes con diagnóstico de cáncer anaplásico de tiroides, intervenidos quirúrgicamente en el Hospital Dr. Gustavo Fricke de Viña del Mar, entre los años 2002 a 2008. Se incluyó la totalidad de los pacientes.

Se registraron características generales, tipo de manejo, características quirúrgicas, hallazgos intraoperatorios y evolución durante la hospitalización. Se presenta la información de cada caso en particular. La información respecto a sobrevida se obtuvo en el Registro Civil e Identificación de Chile.

La información fue analizada en el software Stata 10. Las variables cualitativas se presentan como frecuencias absolutas y las cuantitativas como promedios. La sobrevida se analizó mediante curvas de sobrevida de Kaplan Meier.

\section{Resultados}

Se encontraron 6 casos durante el período de estudio, correspondiendo a 4 hombres y 2 mujeres. La edad promedio fue de 59 años, con un rango de 46 a 84 años. En la Tabla 1 se detallan características generales.

Del total de casos de CAT, en 5 pacientes se realizó cirugía como única terapia y 1 caso complementado con $\mathrm{I}^{131}$. Cuatro de ellas se realizaron con intención curativa. Debido a la extensión local de la neoplasia, 2 pacientes requirieron traqueostomía de forma inmediata. En ninguno de los pacientes se realizó radioquimioterapia pre o postoperatoria. En cuanto a las complicaciones locales de la cirugía sólo un paciente presentó un seroma que fue resuelto de manera conservadora, y de las complicaciones sistémicas un paciente padeció hipocalcemia que se corrigió con tratamiento oral. Un paciente falleció en el postoperatorio mediato debido a complicaciones de la traqueostomía. De los restantes 5 pacientes, la sobrevida promedio fue de 108 días, con sólo 2 pacientes que superan los 6 meses, y ninguno los 10 meses post cirugía (Figura 1).

\section{Caso 1}

Hombre de 46 años, sin antecedentes mórbidos conocidos. Presenta masa cervical de rápido crecimiento adherida a estructuras adyacentes, de $6 \times 5$ $\mathrm{cm}$, asociada a disfagia, dolor, limitación de la movilidad cervical y adenopatías cervicales. Se realiza ecografía (ECO) tiroidea que objetiva tumor sólido lóbulo izquierdo tiroideo y 2 adenopatías cervicales izquierdas de $2 \mathrm{~cm}$; cintigrama tiroídeo informa nódulo hipocaptante izquierdo. Punción aspirativa con aguja fina (PAAF) revela carcinoma de tiroides po- 
Tabla 1. Tratamiento efectuado a 6 pacientes con carcinoma anaplásico de tiroides, Hospital Dr. Gustavo Fricke, Viña del Mar

\begin{tabular}{|c|c|c|}
\hline Género & $\begin{array}{l}\text { Masculino } \\
\text { Femenino }\end{array}$ & $\begin{array}{l}4 \\
2\end{array}$ \\
\hline Edad promedio & & 59 años \\
\hline Origen & $\begin{array}{l}\text { De novo } \\
\text { Papilar }\end{array}$ & $\begin{array}{l}4 \\
2\end{array}$ \\
\hline Cirugía & $\begin{array}{l}\text { Tiroidectomía total bilateral } \\
\text { Lobectomía derecha } \\
\text { Resección local recidiva }\end{array}$ & $\begin{array}{l}4 \\
1 \\
1\end{array}$ \\
\hline Abordaje & Cervical anterior transversa & 6 \\
\hline Disección ganglionar & $\begin{array}{l}\text { Funcional } \\
\text { Radical }\end{array}$ & $\begin{array}{l}5 \\
1\end{array}$ \\
\hline Traqueostomía & $\begin{array}{l}\text { No } \\
\text { Sí }\end{array}$ & $\begin{array}{l}4 \\
2\end{array}$ \\
\hline Duración promedio cirugía & 4,7 horas & \\
\hline Biopsia rápida intraoperatoria & $\begin{array}{l}\text { Sí } \\
\text { No }\end{array}$ & $\begin{array}{l}2 \\
4\end{array}$ \\
\hline Intención & $\begin{array}{l}\text { Curativa } \\
\text { Paliativa }\end{array}$ & $\begin{array}{l}4 \\
2\end{array}$ \\
\hline Radioquimioterapia & No & 6 \\
\hline Radioyodo & $\begin{array}{l}\text { Sí } \\
\text { No }\end{array}$ & $\begin{array}{l}1 \\
5\end{array}$ \\
\hline Estadía promedio & & 6 días \\
\hline Sobrevida promedio & & 108 días \\
\hline
\end{tabular}

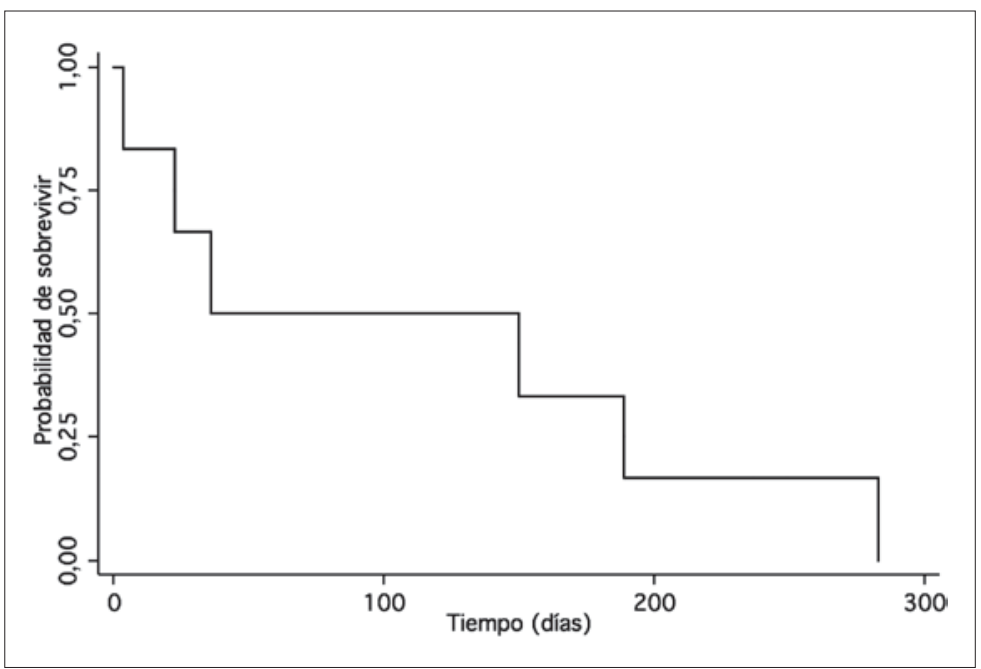

Figura 1. Sobrevida de pacientes operados por carcinoma anaplásico de tiroides, Hospital Dr. Gustavo Fricke, Viña del Mar. bremente diferenciado. Tomografía computada (TC) de tórax informa presencia de metástasis mediastínicas. Nasolaringofibroscopia no objetiva compromiso de vía aérea. Durante la cirugía se encuentra lóbulo tiroideo izquierdo pétreo de $6 \times 5 \mathrm{~cm}$ con adenopatías tumorales en región pretiroidea, pedículo inferior $\mathrm{y}$ triángulo posterior izquierdo. En el postoperatorio mediato, el paciente presenta hipocalcemia sintomática de $6,5 \mathrm{mg} / \mathrm{dL}$ que se corrige con calcio oral. Tres meses después de la cirugía ingresa por síndrome emético y déficit de volumen circulante efectivo, objetivándose hepatomegalia. Fallece a los 184 días post cirugía. 


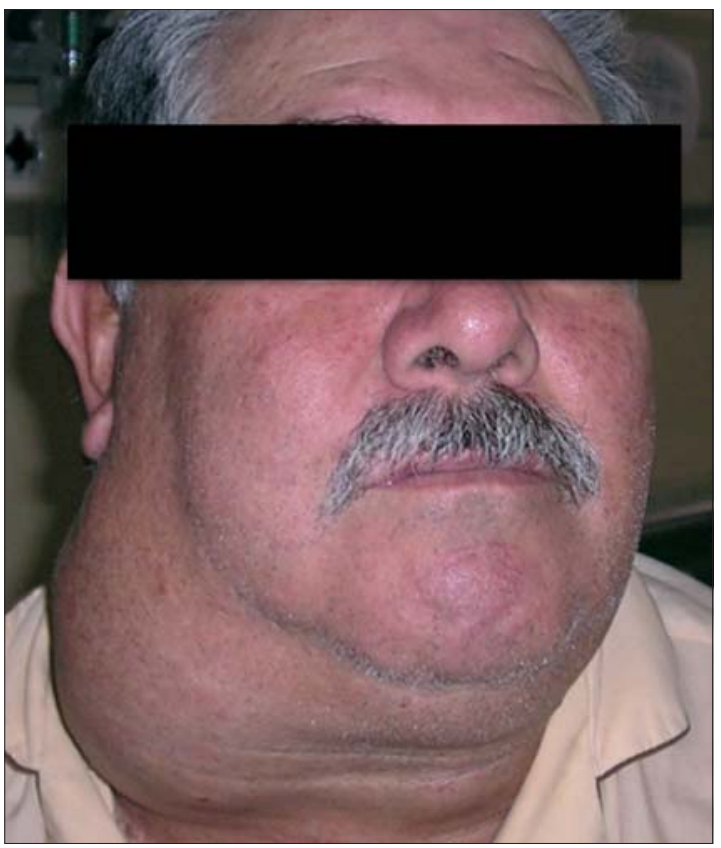

Figura 2. Paciente con cáncer anaplásico de tiroides previo a la cirugía.

\section{Caso 2}

Hombre de 56 años con antecedente de hipertensión arterial crónica (HTA), diabetes mellitus tipo 2 (DM2), tabaquismo activo y masa cervical anterior de 21 años de evolución con rápido crecimiento en los últimos 3 meses, alcanzando diámetro de $20 \mathrm{~cm}$, asociada a disfonía, disfagia y adenopatías cervicales derechas (Figura 2). ECO tiroidea informa masa en lóbulo derecho y adenopatías cervicales derechas. PAAF de adenopatías informa presencia de cáncer indiferenciado. TC de Tórax informa bocio compresivo sin lesión pulmonar. TC de cerebro y ecografía de abdomen sin lesiones. Durante cirugía se objetiva masa tumoral de lóbulo derecho, pétrea, indistinguible de esófago y tráquea, y adenopatías tumorales de cadenas yugular, triángulo posterior derecho y bifurcación carotídea, quedando tumor residual en bifurcación carotídea, tráquea y esófago. Sin complicaciones en el postoperatorio. Fallece a los 36 días post operado.

\section{Caso 3}

Hombre de 50 años con antecedente de DM2. Consulta por masa cervical anterior de $6 \mathrm{~cm}$ de diámetro de 1 mes de evolución, dura, adherida a estructuras adyacentes, asociada a disfagia, disnea, disfonía, estridor leve, dolor cervical progresivo y adenopatías yugulares derechas. ECO muestra masa tumoral en relación a tiroides y adenopatías cervi- cales bilaterales. PAAF de adenopatías informa carcinoma indiferenciado de tiroides. TC cervical objetiva bocio endotorácico, adenopatías yugulares internas bilaterales, abultamiento cuerda vocal izquierda, disminución calibre traqueal. Durante la cirugía se objetiva gran bocio endotorácico pétreo, con compromiso de ambos polos inferiores, vasos cervicales y esófago; firmemente adherida a pared anterior de la tráquea y múltiples adenopatías en ambas cadenas yugulares, realizando traqueostomía. Evoluciona tórpidamente, falleciendo 4 días post cirugía por paro cardiorrespiratorio.

\section{Caso 4}

Mujer de 74 años con antecedente de HTA y lobectomía izquierda de tiroides por carcinoma papilar de tiroides infiltrante a tráquea 6 meses antes. Consulta por masa cervical anterior en relación a lóbulo derecho remanente, de rápido crecimiento, adherida a estructuras adyacentes y adenopatías yugulares derechas. TC cervical objetiva masa tumoral de $6 \mathrm{~cm}$ en relación a lóbulos derecho e izquierdo, adherido a esternocleidomastoídeo, con múltiples adenopatías en cadena yugular interna derecha. Cintigrafía tiroidea objetiva bocio multinodular. Se concluye recidiva de cáncer de lóbulo izquierdo de tiroides hacia lóbulo derecho. Durante cirugía se objetiva masa sólido-quística desde cara anterior de tráquea a región de carótida derecha; vena yugular interna derecha comprometida por tumor tiroideo con zonas nodulares escasas y adenopatías múltiples infratiroideas derechas. Se completó la tiroidectomía. No se presentan complicaciones en el postoperatorio. Fallece a los 115 días post intervención.

\section{Caso 5}

Mujer de 82 años con antecedentes de HTA y bocio de 30 años de evolución. Consulta por dolor cervical derecho y rápido crecimiento de bocio en 15 días, asociado a disnea progresiva, disfagia, disfonía y adenopatías yugulares derechas. Se realiza TC de Cuello y Tórax que objetiva bocio intratorácico con predominio de lóbulo derecho, desplazamiento y reducción de lumen traqueal cercano a carina, adenopatías yugulares derechas y nódulos en ápices pulmonares. En la cirugía se observa un gran bocio bilateral y múltiples adenopatías cervicales. En postoperatorio inmediato presenta falla respiratoria severa, requiriendo traqueostomía por parálisis de cuerdas vocales. Biopsia diferida arroja CAT. Fallece a los 23 días post cirugía.

\section{Caso 6}

Hombre de 48 años con antecedente de tiroidectomía bilateral total y disección ganglionar izquier- 
da por cáncer papilar de tiroides con compromiso de músculo cricofaríngeo izquierdo y nervio recurente izquierdo. Recibió $400 \mathrm{mCu}$ de $\mathrm{I}^{131}$ repartidas en 4 dosis postoperatorias. Durante rastreo con radioyodo, 4 años después, se detecta captación en lecho tiroideo, recibiendo nueva dosis de $\mathrm{I}^{131}$. Seis meses después, se encuentran adenopatías submaxilares y supraclaviculares izquierdas; PAAF demostró carcinoma papilar poco diferenciado del tiroides. TC de tórax sin lesiones. Ingresa con diagnóstico de cáncer papilar con recidiva ganglionar; en cirugía se objetiva que tumor infiltraba esófago, vena yugular interna y nervio vago izquierdos. Se realiza nueva disección ganglionar funcional izquierda. Evoluciona con parálisis de cuerda vocal izquierda y de nervio espinal accesorio izquierdo. La biopsia demostró carcinoma papilar poco diferenciado con metástasis ganglionares y compromiso de bordes quirúrgicos. Recibió $200 \mathrm{mCu}$ de $\mathrm{I}^{131}$ al mes de post operado. Rastreo posterior demostró captación cervical anterior baja paramediana izquierda y se propone nueva dosis de $\mathrm{I}^{131}$. A los 7 meses de post operado ingresa para gastrostomía por afagia. Un mes después reingresa por falla respiratoria severa, falleciendo a los 283 días post intervención.

\section{Discusión}

El diagnóstico del CAT se fundamenta en sospecha clínica, anamnesis, examen físico y exámenes complementarios. Dentro de éstos, es de importancia la realización de PAAF. Estudios han reportado su utilidad como examen preoperatorio para el diagnóstico y manejo de estos pacientes ${ }^{7,13}$. Además la realización de TC de cuello y mediastino permite evaluar la extensión local e invasión de estructuras adyacentes $^{14}$. El uso de resonancia magnética es de utilidad para determinar invasión ósea y vascular, al momento de planificar la resolución quirúrgica ${ }^{15}$.

Las opciones de tratamiento incluyen cirugía, quimioterapia y radioterapia. Se ha reportado que la utilización de terapia unimodal se asocia con menor sobrevida ${ }^{14,16}$, siendo la terapia multimodal de elección actualmente evitando la muerte por invasión local y mejorando la sobrevida. Aún existe controversia en cuanto a la secuencia de utilización de cada terapia. En cuanto a la cirugía, sólo en 13,7\% a $22 \%$ de los pacientes es posible realizar una resección macroscópica con intención curativa sin tratamiento neoadyuvante, dado el patrón de crecimiento local del $\mathrm{CAT}^{17,18}$. La radioquimioterapia neoadyuvante ha sido planteada como la mejor opción para pacientes en que no se puede realizar cirugía inicial con intención curativa ${ }^{19}$, ya que en ellos la resección macroscópica del tumor aumenta a un $92 \%{ }^{16}$ posterior a su uso. También se ha planteado que el uso de cirugía citorreductiva seguida por radioquimioterapia adyuvante representaría una buena opción terapéutica en este grupo de pacientes, con un 56\% de sobrevida a los 6 meses de seguimiento ${ }^{17,20}$. A pesar de lo anterior, al momento del diagnóstico, el $48 \%$ de los pacientes presentan una enfermedad fuera del alcance de tratamiento debido a la presencia de enfermedad local o sistémica avanzada, o de contraindicación para la realización de radioquimioterapia ${ }^{9}$. En ellos, la utilización de cirugía paliativa, como por ejemplo, la instalación de traqueostomía en esta serie, es de gran importancia para el alivio de la sintomatología y la mejora en la calidad de vida.

La sobrevida de los pacientes afectados por CAT es de $31,6 \%$ y $19,3 \%$ a 6 y 12 meses de seguimiento respectivamente ${ }^{11}$. Los factores relacionados a mejor sobrevida son edad menor a 60 años, velocidad de crecimiento del tumor, extensión de la enfermedad local o a distancia, tipo y respuesta a tratamiento realizado ${ }^{11,21}$. De hecho, aquellos pacientes que responden a quimioterapia tienen una mediana de sobrevida mayor en comparación a aquellos pacientes que no responden (32 y 7 meses respectivamente $)^{22}$.

En la serie presentada, el diagnóstico de CAT, se realizó en estados avanzados de la enfermedad en todos los casos, con extenso compromiso local y de órganos adyacentes, dada su capacidad de invasión y crecimiento rápido, lo que refuerza la necesidad de una mayor sospecha clínica y derivación inmediata, permitiendo así la posibilidad de uso de terapias multimodales para su manejo, lo cual no se observó en esta serie. Además, destaca la gran letalidad del CAT en este grupo de pacientes, con una sobrevida observada menor a la reportada a la literatura. Es de esperar que nuevos reportes de pacientes tratados bajo nuevos protocolos informen mejores resultados.

\section{Referencias}

1. Cornett WR, Sharma AK, Day TA, Richardson MS, Hoda RS, van Heerden JA, et al. Anaplastic thyroid carcinoma: an overview. Curr Oncol Rep 2007; 9: 152158.

2. Are C, Shaha AR. Anaplastic thyroid carcinoma: biology, pathogenesis, prognostic factors, and treatment approaches. Ann Surg Oncol 2006; 13: 453 464. Epub 2006 Feb 15.

3. Chiacchio S, Lorenzoni A, Boni G, Rubello D, Elisei R, Mariani G. Anaplastic thyroid cancer: prevalence, diagnosis and treatment. Minerva Endocrinol 2008; 33: $341-357$. 
4. Ain K. Anaplastic Thyroid Carcinoma: Behavior, Biology, and Therapeutic Approaches. Thyroid 1998; 8: 715-726.

5. Venkatesh YS, Ordonez NG, Schultz PN, Hickey RC, Goepfert H, Samaan NA. Anaplastic carcinoma of the thyroid. Cancer 1990; 66: 321-330.

6. Bakiri F, Djemli FK, Mokrane LA, Djidel FK. The relative roles of endemic goiter and socioeconomic development status in the prognosis of thyroid cancinoma. Cancer 1998; 82: 1146-1153.

7. Albores-Saavedra J, Henson DE, Glazer E, Schwartz AM. Changing patterns in the incidence and survival of thyroid cancer with follicular phenotype--papillary, follicular, and anaplastic: a morphological and epidemiological study. Endocr Pathol 2007; 18: 1-7.

8. Salvatore G, Nappi T, Salerno P, Jiang Y, Garbi C, Ugolini C, et al. A Cell Proliferation and Chromosomal Instability Signature in Anaplastic Thyroid Carcinoma. Cancer Res 2007; 67: 10148-10158.

9. McIver B, Hay ID, Giuffrida D, Dvorak CE, Grant CS, Thompson GB, et al. Anaplastic thyroid carcinoma: A 50-year experience at a single institution. Surgery 2001; 130: 1028-1034.

10. Rosen IB, Asa SL, Brierley JD. Anaplastic carcinoma of the thyroid gland. In: Clark OH, Duh QY, Kebebew E, editors. Textbook of Endocrine Surgery. Philadelphia: Elsevier Saunders; 2005. pp 159-167.

11. Kebebew E, Greenspan FS, Clark OH, Woeber KA, McMillan A. Anaplastic thyroid carcinoma. Treatment outcome and prognostic factors. Cancer 2005; 103: 1330-1335.

12. Ain KB. Anaplastic thyroid carcinoma: A therapeutic challenge. Semin Surg Oncol 1999; 16: 64-69.

13. Luze T, Totsch M, Bangerl I, Hittmair A, Sandbichler $\mathrm{P}$, Ladurner D, et al. Fine needle aspiration cytodiagnosis of anaplastic carcinoma and malignant haemangioendothelioma of the thyroid in an endemic goitre area. Cytopathology 1990; 1: 305-310.

14. Mitchell G, Huddart R, Harmer C. Phase II evaluation of high dose accelerated radiotherapy for anaplastic thyroid cancer. Radiother Oncol 1999; 50: 33-38.

15. Pasieka JL. Anaplastic thyroid cancer. Curr Opin Oncol 2003; 15: 78-83.

16. Veness MJ, Porter GS, Morgan GJ. Anaplastic thyroid carcinoma: Dismal outcome despite current treatment approach. ANZ J Surg 2004; 74: 559-562.

17. Sugino K, Ito K, Mimura T, Nagahama M, Fukunari $\mathrm{N}$, Kubo A, et al. The important role of operations in the management of anaplastic thyroid carcinoma. Surgery 2002; 131: 245-248.

18. Nilsson O, Lindeberg J, Zedenius J, Ekman E, Tennvall $\mathrm{J}$, Blomgren $\mathrm{H}$, et al. Anaplastic giant cell carcinoma of the thyroid gland: Treatment and survival over a 25 year period. World J Surg 1998; 2: 725-730.

19. Tennvall J, Lundell G, Wahlberg P, Bergenfelz A, Grimelius L, Akerman M, et al. Anaplastic thyroid carcinoma: three protocols combining doxorubicin, hyperfractionated radiotherapy and surgery. British Journal of Cancer 2002; 86: 1848-1853.

20. Brignardello E, Gallo M, Baldi I, Palestini N, Piovesan A, Grossi E, et al. Anaplastic thyroid carcinoma: clinical outcome of 30 consecutive patients referred to a single institution in the past 5 years. Eur J Endocrinol 2007; 156: 425-430.

21. Besic N, Hocevar M, Zgajnar J, Pogacnik A, GrazioFrkovic S, Auersperg M. Prognostic factors in anaplastic carcinoma of the thyroid-a multivariate survival analysis of 188 patients. Langenbecks Arch Surg 2005; 390: 203-208.

22. Ain KB, Egorin MJ, DeSimone PA. Treatment of anaplastic thyroid carcinoma with paclitaxel: Phase 2 trial using ninety-six hour infusion. Collaborative Anaplastic Thyroid Cancer Health Intervention Trials (CATCHIT) Group. Thyroid 2000; 10: 587-594. 